\title{
Intracranial meningioma as primary presentation for an undiagnosed collision metastatic breast cancer: Case report and literature review
}

\author{
ASHRAF FARRAG ${ }^{1,2}$, JAWAHER ANSARI ${ }^{1}$, MUHAMMAD ALI ${ }^{1}$, GHANEM SUNBULI ${ }^{3}$, \\ HASSAN KASSEM $^{4}$ and ABDUL-AZIZ AL HAMAD ${ }^{1}$
}

${ }^{1}$ Department of Oncology, Prince Sultan Military Medical City, Riyadh, Riyadh $11159 ;{ }^{2}$ Clinical Oncology Department, Assiut University Hospitals, Assiut, Assiut 71515; Departments of ${ }^{3}$ Pathology and ${ }^{4}$ Radiology,

Prince Sultan Military Medical City, Riyadh, Riyadh 11159, Kingdom of Saudi Arabia

Received November 20, 2017; Accepted February 26, 2018

DOI: $10.3892 / \mathrm{mco} .2018 .1589$

\begin{abstract}
Intracranial metastasis from breast cancer is a relatively common finding, however, the appearance of breast cancer metastasis in a meningioma is very rare. Several cases of tumor-to-tumor metastasis and collision tumors have been reported previously, with meningioma being implicated as the most common benign intracranial neoplasm to harbour the metastasis. Occasionally, the discovery of a tumor-to-meningioma metastasis may herald the diagnosis of an occult primary malignancy. Careful histopathological assessment of the resected meningioma specimen is pivotal to the management of these patients, as this will alter the treatment plan and prognosis considerably. Intracranial meningioma with collision breast cancer as primary presentation of an undiagnosed metastatic breast cancer is extremely rare. The current study presents a case of intracranial meningioma with collision breast cancer as a primary presentation, and reviews the available evidence for this unusual disease entity.
\end{abstract}

\section{Introduction}

Breast cancer represents the second most frequent cause of brain metastases (after lung cancer), diagnosed in approximately $15 \%$ of advanced breast cancer cases $(1,2)$. However, the appearance of breast cancer metastasis in a meningioma is extremely rare. Distinguishing between overt breast cancer intracranial metastasis and metastasis in a meningioma is important as the prognosis for these two entities could be very diverse. The terms 'tumor-to-tumor metastasis' and 'collision

Correspondence to: Dr Ashraf Farrag, Department of Oncology, Prince Sultan Military Medical City, Riyadh, P.O. Box 7897, Riyadh, Riyadh 11159, Kingdom of Saudi Arabia

E-mail: drashraffarrag@yahoo.com

Key words: meningioma, breast cancer, collision tumor, tumor to tumor metastases tumor' have been used often interchangeably in literature to describe cases of intra-meningioma metastasis. The term collision indicates the presence of two histologically distinct tumors occurring concurrently in the same anatomic location with some intermingling. Tumor-to-tumor metastasis definition requires the presence of two distinct histopathological features and the encasement of the metastatic focus with a rim of distinct host tumor tissue.

With regards to breast cancer and meningioma, the majority of previously reported cases have highlighted the presentation of tumor-to-tumor metastasis or collision tumor in patients with a previous history of breast cancer. However, our case has a unique presentation of an intracranial meningioma with collision breast cancer as the primary presentation leading to the diagnosis of metastatic breast cancer. There has been only one other reported case of intrameningioma metastasis as a first clinical manifestation of occult primary breast carcinoma (3).

\section{Case report}

A 57-year-old female presented with a 6-week history of vertigo and headache. Her Glasgow coma scale was 15/15. She had no medical comorbidities and her World Health Organization (WHO) performance status was 1. Magnetic Resonance Imaging (MRI) scan revealed an extra-axial dural-based mass overlying the right lateral sphenoid wing with intense enhancement, dural tailing and perilesional oedema consistent with a meningioma (Fig. 1). She underwent craniotomy and sub-total resection of the suspected right sphenoid wing meningioma. Histopathology revealed two distinct neoplastic processes (Figs. 2 and 3). The first was a WHO grade I meningioma of transitional type with low mitotic activity $[<1$ mitoses/10 high power field (hpf)] and low Ki-67 proliferation index $(<2 \%)$. The second neoplastic process consisted of a malignant tumor composed of corded epithelioid cells with extensive necrosis, very high mitotic activity ( $>30$ mitoses/10 hpf) and a high Ki-67 proliferation index $(>40 \%)$. Immunohistochemical (IHC) studies for the grade I meningioma component showed positive staining 


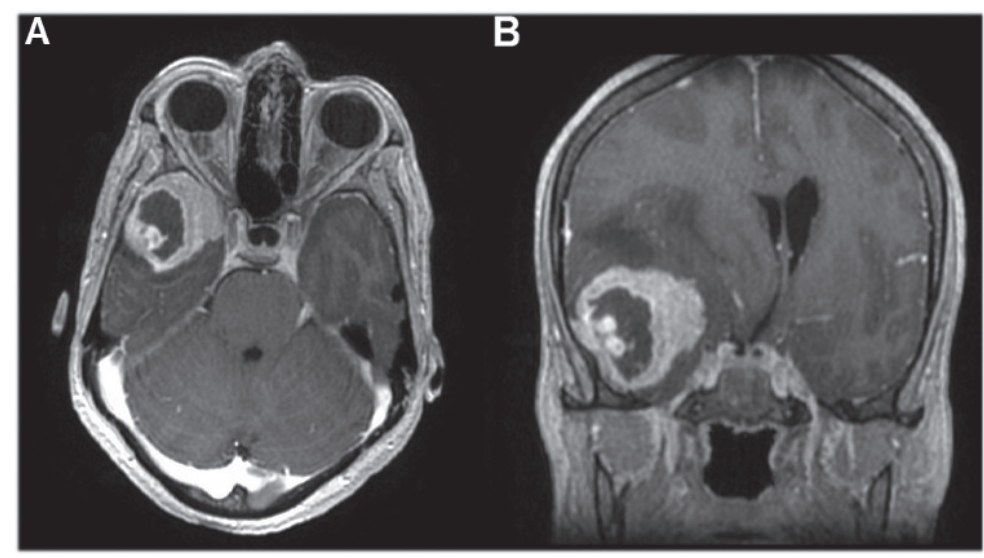

Figure 1. Brain MRI at presentation, post-contrast T1 weighted axial image (A) and coronal image (B) demonstrating the right lateral sphenoid wing meningioma. Both images show non-enhancing area of breaking down containing enhancing mural nodules, which is atypical for meningioma.

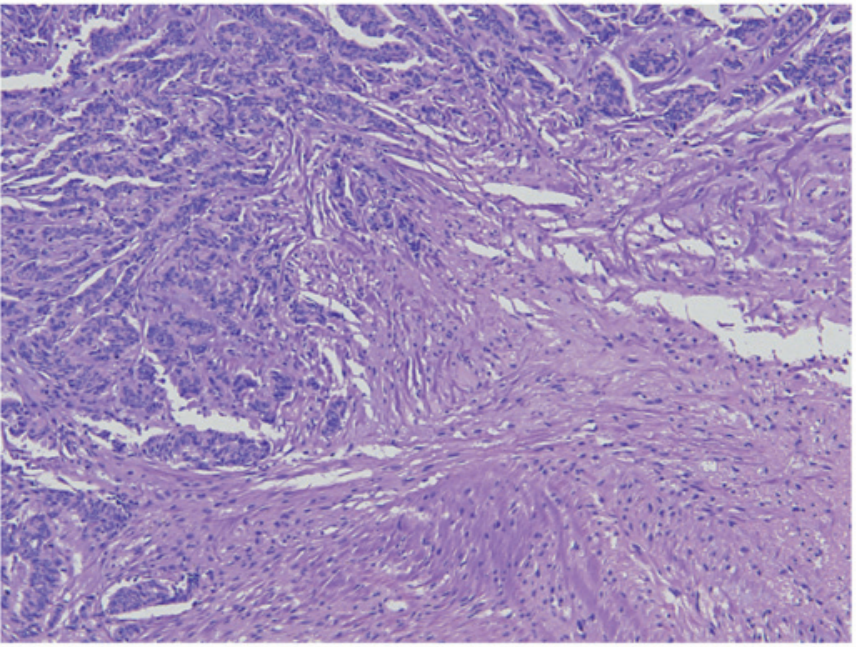

Figure 2. Two distinct neoplastic processes are shown. First one (right lower) composed of meningeothelial cells with fibrous stroma and second one (left upper) consists of malignant epithelial cells with necrosis (magnification, $\mathrm{x} 10$ ).

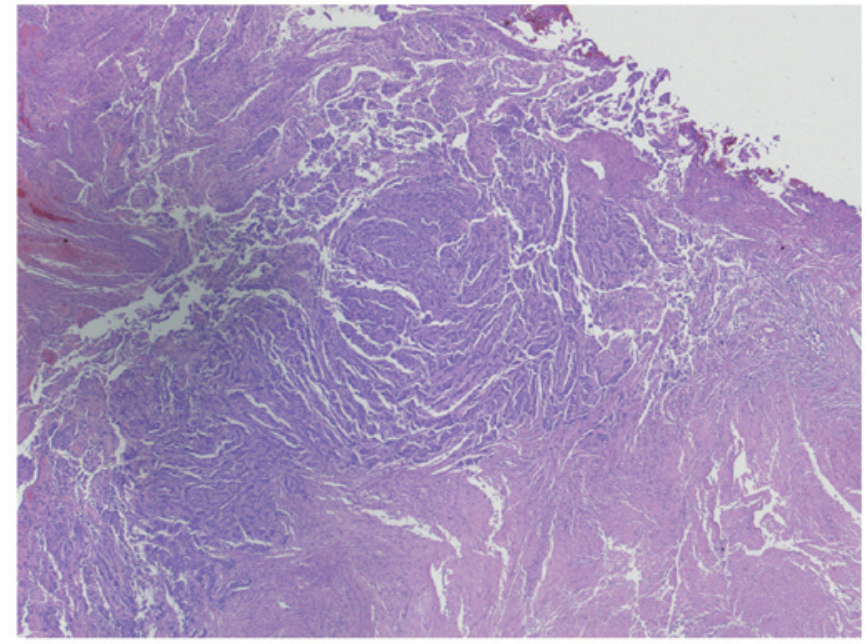

Figure 3. The malignant epithelial cell of breast cancer (in the middle) surrounded in both sides by the meningeothelial cell of meningioma is shown (magnification, x10). for epithelial membrane antigen (EMA) and progesterone receptor $(\mathrm{PR})$ nuclear expression. IHC studies for the malignant tumor showed positive staining for EMA, but negative oestrogen Receptor (ER), PR and pan-cytokeratin. HER 2 Fluorescence in situ hybridization (FISH) was negative. The findings favoured the likely diagnosis of WHO grade 3 meningioma, however, alluded to the possibility of a metastatic collision tumor provided there was evidence of a concomitant malignancy.

Post-operative clinical examination revealed a suspicious right breast mass. Mammography and breast ultrasound revealed a highly suspicious lesion in the right breast associated with axillary lymphadenopathy. Tru-cut biopsy from the suspicious right breast lesion showed mucinous carcinoma of the breast with positive IHC for ER (>90\%) and PR (15\%). Staging isotope bone scan and computed tomography (CT) scan for chest, abdomen and pelvis showed multiple bone metastases but no visceral metastases. MRI spine showed multiple spinal metastases with spinal cord compression at thoraco-lumbar spine (T12-L1 level). She underwent posterior decompression and spinal fixation followed by post-operative palliative spinal radiotherapy 20 Gray in 5 fractions. Histopathology from the bone biopsy confirmed the presence of metastatic adenocarcinoma cells consistent with metastatic breast cancer. IHC profile was negative for ER, PR and cytokeratin (CK)-20 but was positive for CK-7. She was considered for cranial irradiation but following a discussion at the multidisciplinary tumor board meeting it was decided that further re-resection of the cranial lesion should be the preferred approach if the lesion increased in size or the patient became symptomatic during follow-up.

She was commenced on systemic endocrine therapy with oral letrozole along with monthly zoledronic acid infusions. Seven months later, she developed disease progression with worsening axillary lymphadenopathy and new bone metastases. MRI brain showed increase in the size and extent of the previously noted right middle cranial fossa extra-axial dural-based space occupying lesions along with oedema and midline shift suggestive of disease progression. She underwent craniotomy and complete resection of the right fronto-temporal lesion. Histopathology revealed a malignant neoplasm with features similar to the patient's previous breast tumor biopsies, although the mucinous component was lacking, and the IHC panel 
showed negative expression for ER, PR and pan-cytokeratin. Overall features were suggestive of a collision metastatic breast cancer involving a grade 1 meningioma. She was offered whole brain radiotherapy but she declined this as she was concerned regarding hair loss and possibility of cognitive deterioration. She was treated with capecitabine chemotherapy for 10 months followed by second-line endocrine-based treatment with a combination of everolimus and letrozole on further disease progression. Follow-up MRI brain performed recently has not shown any evidence of disease recurrence. She remains clinically and radiologically stable on her current systemic treatment 3 years on from her initial presentation.

In summary, this lady's presentation with a collision breast tumor involving a low-grade meningioma led to the diagnosis of metastatic breast cancer. Following surgical treatment of her intra-cranial disease and spinal cord decompression, she remains stable on systemic endocrine therapy 3-years on following her initial presentation.

\section{Discussion}

Several cases of tumor-to-tumor metastases and collision tumors have been reported previously (4-9). Based on case series and retrospective studies, the most frequent donor tumor appears to be lung carcinoma and the most common malignant recipient is renal cell carcinoma $(10,11)$. Meningioma appears to be the most common benign recipient tumor (12). The process of epithelial-mesenchymal transition (EMT) is thought to enable cancer cells to acquire less adhesion and more motility enhancing their ability to migrate leading to tumor metastases and progression (13). Primary tumor-derived components, tumor-mobilized bone- marrow-derived cells (BMDCs), and the local stromal microenvironment of the host are the three major factors crucial for the formation of the pre-metastatic niche. The pre-metastatic niche can be defined as the supportive and receptive microenvironment in the host tissue that undergoes a series of molecular and cellular changes to help for the subsequent seeding and colonization of tumor cells (14).

The association between breast cancer and meningioma is controversial. Early reports observed a strong epidemiological association between breast cancer and meningioma suggesting that women with either condition had a higher risk of being diagnosed with the other condition (15). Meningiomas are twice as common in women than men, and, like breast cancer, have a predilection for the fifth or sixth decades of life and similarly tend to grow during pregnancy (16). However, several retrospective cohort studies have shown conflicting results regarding the association between breast cancer and meningioma (17-19). More recently, it has also been questioned whether the presumed association between breast cancer and meningioma could simply be related to the increased frequency of cranial imaging for staging and/or follow-up, particularly among women with advanced stage breast cancer (20). Several factors may contribute to the development of metastases in a meningioma including, high vascularity, slow growth and hormonal influences (21). The highly collagenous and vascular histology of meningiomas, combined with its slow growth rate for a prolonged duration provides a fertile environment for development of intra-tumoral metastases (22). The mutual expression of E-cadherin may facilitate the seeding of one tumor by another (23). Amplification of c-myc oncogene may play a role in estrogen-induced proliferation and in the pathogenesis of both breast cancer and meningioma (24).

Certain criteria were proposed for the diagnosis of tumorto-tumor metastasis. Mainly, there must be an evidence of at least two primary tumors and the recipient tumor must be a true neoplasm. Direct contiguous growth or tumor emboli from an adjacent tumor are excluded; and the recipient cannot be a lymph node involved by leukemia or lymphoma (25). In addition, Pamphlett et al proposed additional criteria for the diagnosis of true tumor-to-meningioma metastasis: the metastatic focus must at least be partially enclosed by a rim of histologically distinct host tumor tissue; and the existence of the metastasizing primary carcinoma must be proven and compatible with the metastasis (26).

Routine radiological imaging techniques such as $\mathrm{CT}$ or MRI cannot reliably exclude the presence of metastasis within a meningioma, however, perfusion MRI and MR spectroscopy provide additional functional assessment and are likely to provide additional diagnostic information (27). The limitations of radiological diagnosis of this unusual lesion underscore the importance of careful pathologic analysis as the diagnosis of breast carcinoma- to-meningioma metastasis can be missed if the entire tumor is not systematically sampled. This merits careful coordination between surgeons and pathologists in cases where tumor- to-tumor metastasis is possible, given its potential implications for patient prognosis and subsequent management (28).

In our case, the diagnosis of the breast cancer collision tumor in the meningioma led to further assessment and diagnosis of high-risk metastatic breast cancer with spinal cord compression. Prompt diagnosis of breast cancer followed by surgery and radiotherapy prevented catastrophic and debilitating consequences such as paraplegia.

In conclusion, this case highlights the rare presentation of a metastatic collision breast carcinoma with primary presentation mimicking a high-grade meningioma. It is important to be aware about this unusual condition as careful pathologic analysis of the resected meningioma, high index of suspicion for breast cancer, and prompt intervention prevented significant morbidity in this case.

\section{Acknowledgements}

Not applicable.

\section{Funding}

No funding was received.

\section{Availability of data and materials}

The datasets used in the current article are available from the corresponding author on request.

\section{Authors' contributions}

AF, JA, conception and design. AF, JA, MA, GS, HK, AH wrote, reviewed and gave final approval of the manuscript to be published. JA and AH were study supervisors. 


\section{Competing Interests}

The authors declare they have no competing interests.

\section{Ethics approval and consent to participate}

Not applicable

\section{Consent for publication}

Written informed consent was obtained.

\section{References}

1. Lin NU, Bellon JR and Winer EP: CNS metastases in breast cancer. J Clin Oncol 22: 3608-3617, 2004

2. Barnholtz-Sloan JS, Sloan AE, Davis FG, Vigneau FD, Lai P and Sawaya RE: Incidence proportions of brain metastases in patients diagnosed (1973 to 2001) in the Metropolitan Detroit Cancer Surveillance System. J Clin Oncol 22: 2865-2872, 2004.

3. Caroli E, Salvati M, Giangaspero F, Ferrante L and Santoro A: Intrameningioma metastasis as first clinical manifestation of occult primary breast carcinoma. Neurosurg Rev 29: 49-54, 2006.

4. Fadare O, Parkash V,Fiedler PN, Mayerson AB and Asiyanbola B: Tumor-to-tumor metastasis to a thyroid follicular adenoma as the initial presentation of a colonic adenocarcinoma. Pathol Int 55: 574-579, 2005.

5. Sayegh ET, Burch EA, Henderson GA, Oh T, Bloch O and Parsa AT: Tumor-to-tumor metastasis: Breast carcinoma to meningioma. J Clin Neurosci 22: 268-274, 2015.

6. Bohn OL, De las Casas LE and Leon ME: Tumor-to-tumor metastasis: Renal cell carcinoma metastatic to papillary carcinoma of thyroid-report of a case and review of the literature. Head Neck Pathol 3: 327-330, 2009.

7. Takei H and Powell SZ: Tumor-to-tumor metastasis to the central nervous system. Neuropathology 29: 303-308, 2009.

8. Basil I, Ru K, Pu C, Silverman J and Jasnosz K: A collision tumor: Primary central nervous system B-cell lymphoma and anaplastic astrocytoma. Lab Med 42: 324-328, 2011.

9. Greer WS, Gardner JM and Montgomery CO: Collision tumor of bone: Primary chondrosarcoma of bone as a rare recipient of tumor-to-tumor metastasis from metastatic breast carcinoma. Case Rep Clin Pathol 2: 25-29, 2015.

10. Ichijima K, Yamabe H, Kobashi Y and Iwata T: Metastasis of cancer to cancer. Acta Pathol Jpn 30: 293-300, 1980.

11. Sella A and Ro JY: Renal cell cancer: Best recipient of tumor-totumor metastasis. Urology 30: 35-38, 1987.

12. Honma K, Hara K and Sawai T: Tumour-to-tumour metastasis. A report of two unusual autopsy cases. Virchows Arch A Pathol Anat Histopathol 416: 153-157, 1989.

13. Thiery JP: Epithelial-mesenchymal transitions in tumour progression. Nat Rev Cancer 2: 442-454, 2002.

14. Liu Y and Cao X: Characteristics and Significance of the Pre-metastatic Niche. Cancer Cell 30: 668-681, 2016.
15. Schoenberg BS, Christine BW and Whisnant JP: Nervous system neoplasms and primary malignancies of other sites. The unique association between meningiomas and breast cancer. Neurology 25: 705-712, 1975

16. Smith FP, Slavik M and MacDonald JS: Association of breast cancer with meningioma: Report of two cases and review of the literature. Cancer 42: 1992-1994, 1978.

17. Custer BS, Koepsell TD and Mueller BA: The association between breast carcinoma and meningioma in women. Cancer 94: 1626-1635, 2002

18. Criscitiello C, Disalvatore D, Santangelo M, Rotmensz N, Bazolli B, Maisonneuve P, Goldhirsch A and Curigliano G: No link between breast cancer and meningioma: Results from a large monoinstitutional retrospective analysis. Cancer Epidemiol Biomarkers Prev 23: 215-217, 2014.

19. Cea-Soriano L, Blenk T, Wallander MA and Rodríguez LA: Hormonal therapies and meningioma: Is there a link? Cancer Epidemiol 36: 198-205, 2012.

20. Milano MT and Grossman CE: Meningioma in breast cancer patients: Population-based analysis of clinicopathologic characteristics. Am J Clin Oncol 40: 11-16, 2017.

21. Okada E, Nakamura M, Koshida Y, Mukai K, Toyama Y and Matsumoto M: Breast carcinoma metastasis to meningioma in the thoracic spine: A case report and review of the literature. J Spinal Cord Med 38: 231-235, 2015.

22. Watanabe T, Fujisawa H, Hasegawa M, Arakawa Y, Yamashita J, Ueda F and Suzuki M: Metastasis of breast cancer to intracranial meningioma: Case report. Am J Clin Oncol 25: 414-417, 2002.

23. Shimada S, Ishizawa K and Hirose T: Expression of E-cadherin and catenins in meningioma: Ubiquitous expression and its irrelevance to malignancy. Pathol Int 55: 1-7, 2005.

24. Leeman DJ, Chandrasekhar SS, Brackmann DE and Poletti BJ: Collision tumors at the cerebellopontine angle: Case report with literature review. Otolaryngol Head Neck Surg 117: S76-S80, 1997.

25. Campbell LV Jr, Gilbert E, Chamberlain CR Jr and Watne AL: Metastases of cancer to cancer. Cancer 22: 635-643, 1968.

26. Pamphlett R: Carcinoma metastasis to meningioma. J Neurol Neurosurg Psychiatry 47: 561-563, 1984.

27. Jun P, Garcia J, Tihan T, McDermott MW and Cha S: Perfusion MR imaging of an intracranial collision tumor confirmed by image-guided biopsy. AJNR Am J Neuroradiol 27: 94-97, 2006.

28. Sayegh ET, Henderson GA, Burch EA, Reis GF, Cha S, Oh T, Bloch $\mathrm{O}$ and Parsa AT: Intrameningioma metastasis of breast carcinoma. Rare Tumors 6: 5313, 2014. 